\title{
Comparison of Polymyxin E and Polymyxin $B$ as an Additive to Pulmonary Surfactant in Escherichia coli Pneumonia of Ventilated Neonatal Rabbits
}

\author{
Guido Stichtenoth $^{\mathrm{a}, \mathrm{b}} \quad$ Marie Haegerstrand-Björkman ${ }^{\mathrm{b}} \quad$ Gabi Walter $^{\mathrm{a}}$ \\ Bim Linderholm $^{b}$ Egbert Herting ${ }^{a}$ Tore Curstedt ${ }^{b}$ \\ ${ }^{a}$ Department of Pediatrics, University of Lübeck, Lübeck, Germany; ${ }^{b}$ Department of \\ Molecular Medicine and Surgery, Karolinska Institutet at Karolinska University Hospital, \\ Stockholm, Sweden
}

\begin{abstract}
What Is It about?
This study compares topical antibiotic intratracheal treatment between two novel preparations composed of exogenous porcine surfactant plus polymyxin B with addition of polymyxin E using an animal model of neonatal Escherichia coli pneumonia. Lung function, survival, pulmonary bacterial growth, and bacterial translocation are studied. Furthermore, histopathology and proinflammatory IL-8 were compared between the treatment groups.
\end{abstract}

\section{Keywords}

Neonatal pneumonia $\cdot$ Pulmonary surfactant $\cdot$ Polymyxin $\cdot$ Pulmonary inflammation $\cdot$ Rabbit pups

\section{Abstract}

Background: Ascending maternofetal bacterial infections often result in premature birth and neonatal respiratory distress. These neonates are treated with exogenous pulmonary surfactant (SF) and systemic antibiotics. Polymyxins are antimicrobiotic peptides that may bind to SF phospholipids. Objectives: Does topical administration of SF/polymyxin reduce bacterial growth in neonatal rabbit pneumonia and improve pulmonary function? Methods: Neonatal rabbits were tracheotomized and treated intratracheally with mixtures of porcine SF, SF/polymyxin $E(P x E)$, or polymyxin $B(P x B)$. Control animals received saline. Animals were then inoculated with Escherichia coli and ventilated for $4 \mathrm{~h}$. During the experiment, peak insufflation pressures, dynamic lung compliance, and ECG were recorded. Pulmonary and renal bacterial load were determined. Lung histology was performed. Lung and kidney IL-8 were measured in subgroups. Results: Eighty-five animals were included in 2 experimental series, of which 
$78 \%$ survived $4 \mathrm{~h}$ of ventilation. E. coli inoculation caused severe neonatal pneumonia with median IL-8 levels of $2.2 \mathrm{ng} / \mathrm{g}$ in the lungs compared to a median of $0.2 \mathrm{ng} / \mathrm{g}$ in the lungs of the saline controls $(p<0.01)$. Lung compliance after $4 \mathrm{~h}$ was significantly increased at a mean of $0.48 \mathrm{ml} /\left(\mathrm{kg} \cdot \mathrm{cm} \mathrm{H}_{2} \mathrm{O}\right)$ in the SF group and 0.43 in the SF + PxE group compared to 0.35 in the $E$. coli group $(p<0.01)$. In direct comparison, bacterial growth found in the $E$. coli group was reduced 20-fold in the SF + PxB group compared to 75-fold in the SF + PxE group. Conclusion: Addition of polymyxin to SF effectively promotes antimicrobial treatment and improves lung function in neonatal pneumonia of rabbits.

(C) 2017 The Author(s)

Published by S. Karger AG, Basel

\section{Introduction}

Bacterial pneumonia is life-threatening in neonates. Gram-positive group B streptococci and Gram-negative Escherichia coli are the most abundant pathogens in early-onset sepsis [1]. Subsequently to maternal premature rupture of the membranes, ascending bacteria can reach the conducting airways that promote the second largest epithelial surface to the outside environment [2-4].

Due to respiratory distress and risk of infections, neonates are often treated with antibiotics and exogenous surfactant (SF). Epithelial airway damage, SF deficiency, and inflammation may cause a vicious cycle that ultimately ends in perpetuation of respiratory distress, sepsis, and bronchopulmonary dysplasia.

Polymyxins are antibiotics with a cyclic polycationic antimicrobiotic lipopeptide structure [5]. Due to neurotoxic and nephrotoxic side effects, polymyxins are mainly used for topical therapy.

The use of SF as a vector for topical pulmonary drug administration is a concept that has been evaluated for mixtures with antibiotics [6, 7], including polymyxin B (PxB) [8]. Polymyxins and SF-specific proteins B and C have some similar properties. Thus, we hypothesized mutual functional support in polymyxin-SF mixtures.

In the present study, we focused on prophylactic treatment of mixtures composed of SF and polymyxin E (PxE) versus $\mathrm{PxB}$ in neonatal rabbit pneumonia.

\section{Methods}

\section{Surfactant Preparations}

Poractant alpha (SF, Curosurf ${ }^{\circledR}$, Chiesi Farmaceutici, Parma, Italy) is a modified porcine $\mathrm{SF}$ at a phospholipid concentration of $80 \mathrm{mg} / \mathrm{mL}$. PxE sulfate (Colistin sulfate salt, Sigma Aldrich; No. C4461) or PxB sulfate (Sigma Aldrich No. P1004) were suspended in saline at 20 (PxE) or $80 \mathrm{mg} / \mathrm{mL}$ (PxB or PxE) and mixed with SF at a ratio of 1:100. The resulting phospholipid concentrations were $79.2 \mathrm{mg} / \mathrm{mL}$ in the PxB- or PxE-containing preparations which is close to the original SF preparation of $80 \mathrm{mg} / \mathrm{mL}$. The polymyxin concentrations were 0.2 or $0.8 \mathrm{mg} / \mathrm{mL}$.

\section{General Animal Model}

Frozen E. coli aliquots, ATTC 25922, were recultivated for $16 \mathrm{~h}$ in bouillon (7882; Merck, Darmstadt, Germany), subsequently transferred into new bouillon for $1 \mathrm{~h}$ to reach a midlogarithmic growth phase and washed, and then the stock was adjusted to $\sim 10^{8}$ colonyforming units $(\mathrm{CFU}) / \mathrm{mL}$ using a spectrophotometer. A viability count confirmed the specific concentration. 
Pregnant New Zealand White does were kept in an appropriate environment with a sufficient supply of food and water.

At the gestational age of 29 days (term gestation 31 days), the does were anesthetized using diazepam and fluanisone/fentanyl. Up to 9 pups were born by cesarean section, anesthetized using pentobarbital sodium, and tracheotomized. Subsequently, the animals were treated intratracheally, positioned in a warm plethysmograph $\left(37^{\circ} \mathrm{C}\right)$, relaxed by pancuronium bromide, and connected to the ventilator and the ECG. Lungs were opened by 5 inspirations at peak insufflation pressures (PIP) of $35 \mathrm{~cm} \mathrm{H}_{2} \mathrm{O}$, a frequency of $40 / \mathrm{min}$, and an inspiration:expiration ratio of $1: 3$ at $100 \%$ oxygen. PIP was reduced corresponding to individual tidal volumes of 6-7 mL/kg body weight (bw). Plethysmographic individual tidal volumes and PIP were recorded before inoculating bacteria (15 min), at $30 \mathrm{~min}$, and then every $30 \mathrm{~min}$ until $4 \mathrm{~h}$. Compliance was calculated by dividing the tidal volume by the corresponding PIP and the body weight. Death was considered when ECG showed heart frequencies below $100 \mathrm{bpm}$. After $2 \mathrm{~h}$ of additional pentobarbital, pancuronium bromide and glucose $10 \%$ were given.

\section{Experimental Series}

We performed 2 experimental series. The SF dose was $200 \mathrm{mg} / \mathrm{kg}$ bw. The "PxE series" was comprised of 5 treatment groups: the saline control and E. coli groups received $2.5 \mathrm{~mL} /$ $\mathrm{kg}$ bw saline, and the SF, SF/PxE $1 \%$ and SF/PxE $0.25 \%$ groups received the corresponding volume of each SF preparation. The "PxE versus PxB series" was composed of an E. coli, SF, $\mathrm{SF} / \mathrm{PxE} 1 \%$, and SF/PxB 1\% group at an identical setting. With the exception of the saline control group in the "PxE series," all animals were inoculated with E. coli at 15 min.

\section{Sample Preparation}

Animals were killed by intracerebral injection of lidocaine $2 \%$. The kidneys were excised and the thorax was inspected for evidence of pneumothorax. In the "PxE series," we determined the bacterial load of the left kidney and the right lung. The left lung was processed for histology. In the "PxE versus PxB series," the left main bronchus was tied and the left lung was excised and divided in a central part for histology and a peripheral part for determination of bacterial load. The remaining right lung underwent bronchoalveolar lavage for future analysis.

All samples were weighed.

\section{Histology}

Lungs were fixed for $24 \mathrm{~h}$ in $4 \%$ buffered formalin and embedded in paraffin, and large transversal sections of 3-5 $\mu \mathrm{m}$ thickness were stained with hematoxylin/eosin. Semiquantitatively, alveolar air expansion was estimated by a 5-grade scale $(0,1-25,26-50,51-75$, 76-100\%), airway epithelial necrosis by a 4-grade scale (absent, mild, moderate, prominent), and inflammation and pulmonary hemorrhage by a 3-grade scale (absent, moderate, prominent).

\section{Homogenization}

Samples were washed twice, normalized at a weight of $1 \mathrm{~g}$ (kidney) or $1.5 \mathrm{~g}$ (lung) by adding saline, and homogenized at 30,000 rpm using sterile tips (Omni TH; Omni International, Marietta, GA, USA).

\section{Bacterial Load}

Aliquots of homogenized (left) kidneys and serially diluted lung homogenates were transferred to Petri dishes and mixed with warm Columbia agar base (Oxoid CM 331-550 g) 
containing 5\% defibrinated sheep blood. Dishes were incubated for $24 \mathrm{~h}$ at $37^{\circ} \mathrm{C}$ and the average count from duplicates showing 0-250 CFU/dish was considered for analysis.

\section{Interleukin ELISA}

Homogenates were stored on ice until centrifugation at 3,000 $\mathrm{g}$ for $10 \mathrm{~min}$. IL-8 was determined in supernatants of 42 right lung and 37 right kidney homogenates using a sandwich enzyme immunoassay kit (SEA080Rb; Cloud-Clone Corp., Houston, TX, USA). If needed, samples were diluted in phosphate-buffered saline.

\section{Statistical Analysis}

Statistical analysis was performed using the GraphPad Prism package (4.02; GraphPad Software, La Jolla, CA, USA). For nonparametric bacterial growth and semiquantitatively assessed histology data, Mann-Whitney tests were performed. Survival was compared using $\chi^{2}$ tests. All other data underwent $t$ tests.

\section{Results}

\section{General Observations}

In the "PxE series," 51 neonatal rabbits with a mean birth weight of $41.5 \pm 7.5$ g were obtained ( $n=8-11 /$ group). The mean $E$. coli stock concentration was $4 \times 10^{8} \mathrm{CFU} / \mathrm{mL}$. Three animals that died during initial treatment were excluded. During ventilation, an additional 12 (25\%) animals died. We found no differences in the weight of the right lung.

In the "PxE versus PxB series," 41 pups with a mean birth weight of $44.3 \pm 5.3$ g were treated ( $n=8-10 /$ group). The mean $E$. coli stock concentration was $2 \times 10^{9} \mathrm{CFU} / \mathrm{mL}$. Four animals were excluded from analysis: 2 died during initial treatment, 1 developed pneumothorax, and 1 had tracheal cannula dislocation. During ventilation, 7 animals (18\%) died.

In both series, we found no statistical differences regarding birth weight or survival.

\section{Lung Function}

Overall, dynamic compliance was between 0.36 and $0.55 \mathrm{~mL} / \mathrm{kg} \times \mathrm{cm} \mathrm{H}_{2} \mathrm{O}$ (mean) and tended to decrease during ventilation. However, the differences in lung compliance within each series were too small for providing significance values.

First, a combined analysis of surviving animals from both series revealed an increased dynamic lung compliance (mean $\pm \mathrm{SD}\left[\mathrm{mL} /\left(\mathrm{kg} \cdot \mathrm{cm} \mathrm{H}_{2} \mathrm{O}\right)\right]$ ) after $4 \mathrm{~h}$ of ventilation in SF- $(0.48$ $\pm 0.13)$ and $\mathrm{SF}+$ PxE-treated animals $(0.43 \pm 0.08)$ compared to the E. coli group $(0.35 \pm 0.09$; $p<0.01$ ).

\section{Microbiology}

In the "PxE series," the pulmonary bacterial load of the E. coli and SF groups was similar (median $\log _{10} \sim 8.5 \mathrm{CFU} / \mathrm{g}$ lung). In comparison, treatment with SF $+\mathrm{PxE}(0.25$ or $1 \%$ ) significantly reduced the bacterial load by $2 \log$-steps $(p<0.01$; Fig. 1$)$. A comparison of the bacterial concentration stratified to each experimental day with pups from identical does showed a 33-fold reduction of SF + PxE-treated animals compared to SF-treated pups. Similarly, in the "PxE versus PxB series," the bacterial load of the E. coli and SF groups were 8.8 and $8.5 \mathrm{CFU} / \mathrm{g}$ lung (median $\log _{10}$ ), respectively, which also was significantly reduced by $2-3$ log-steps after addition of $1 \%$ PxB or PxE (Fig. 2). In direct comparison, again stratified to each experimental day using results of pups from identical does, $\mathrm{SF}+\mathrm{PxE}$ treatment gave a 75 -fold reduction of bacteria per g lung compared to a 20 -fold reduction in $\mathrm{SF}+\mathrm{PxB}$-treated animals. 
(C) 2017 The Author(s). Published by S. Karger AG, Basel www.karger.com/bmh

Stichtenoth et al.: Comparison of Polymyxin E and Polymyxin B as an Additive to

Pulmonary Surfactant in Escherichia coli Pneumonia of Ventilated Neonatal Rabbits

Fig. 1. Bacterial load in lungs of the polymyxin E (PxE) series. Scattergram of $\log _{10}$ of the bacterial load in lungs of PxE series after $4 \mathrm{~h}$ of ventilation of neonatal rabbits treated initially with intratracheal surfactant (SF), SF plus $0.25 \%$ or $1 \%$ polymyxin $\mathrm{E}$ (SF/PxE), or saline (saline, Escherichia coli). With the exception of the saline group, all animals were inoculated at $15 \mathrm{~min}$ of ventilation with E. coli. Bars show medians of 8-11 animals per treatment group. ${ }^{* *} p<0.001$ vs. E. coli and SF.

Fig. 2. Bacterial load in lungs of polymyxin E (PxE) versus polymyxin $(\mathrm{PxB})$ series. Scattergram of $\log _{10}$ of the bacterial load in lungs after $4 \mathrm{~h}$ ventilation of neonatal rabbits treated initially with intratracheal surfactant (SF), SF plus $1 \%$ polymyxin E (SF/PxE $1 \% ; \mathrm{w} / \mathrm{w}$ ) or SF plus $1 \%$ polymyxin B (SF/PxB 1\%; w/w), or saline (Escherichia coli). After 15 min of ventilation, all animals received E. coli bacteria. Bars show medians of 7-10 animals per group surviving treatment $>30$ min. ${ }^{* *} p<0.01$ vs. E. coli and SF; ${ }^{\dagger} p<0.05$ vs. SF/PxB $1 \%$.
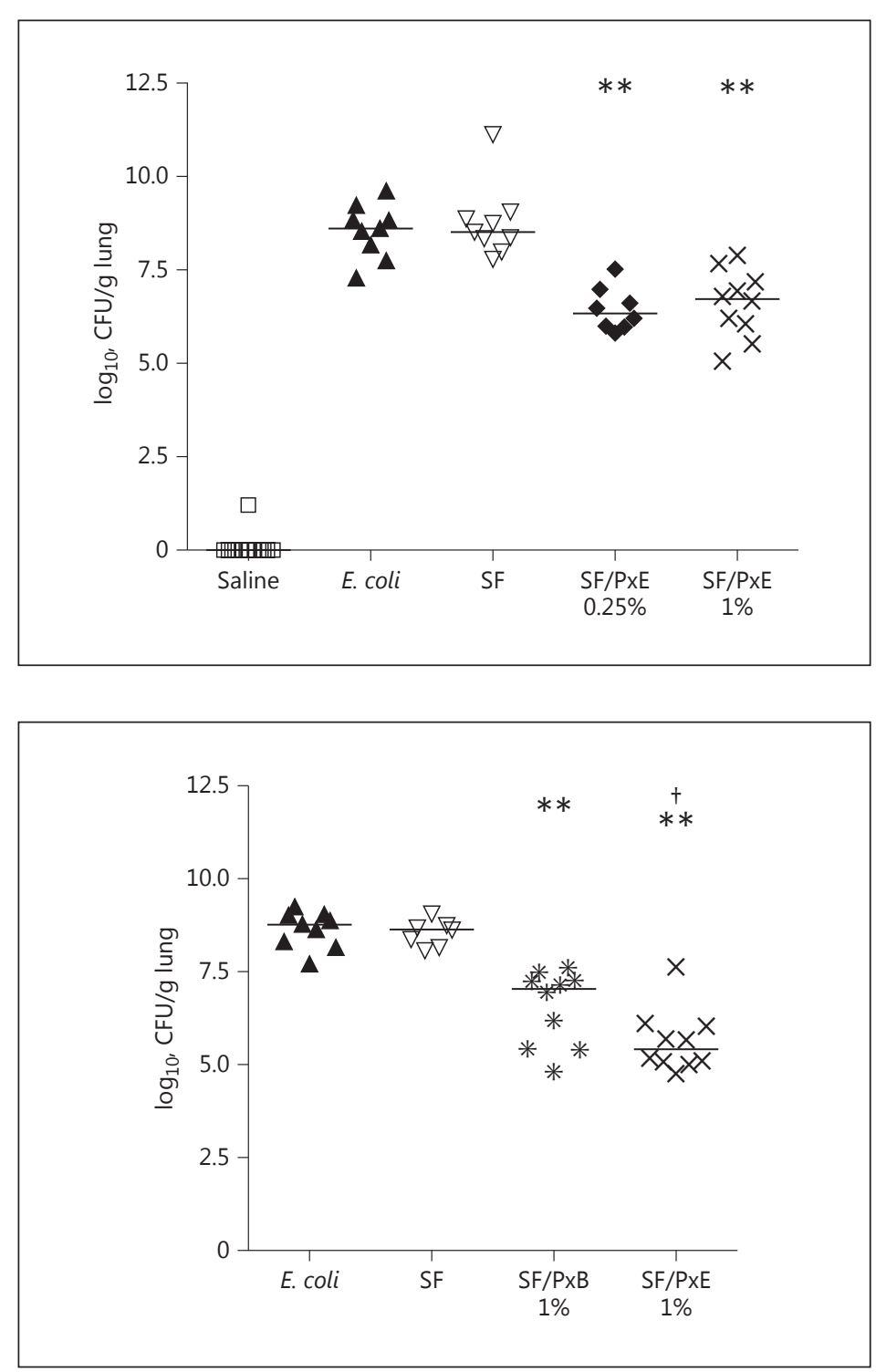

Bacterial translocation to the kidney was found in 8 of 10 surviving animals of the E. coli group in the "PxE series," in 3 of 9 animals treated with SF, and in 2 of 8 animals treated with SF/PxE $0.25 \%$, but only in 1 of 10 animals treated with SF/PxE $1 \%$ ( $p<0.01$ vs. the E. coli group). In the "PxE versus PxB series," we only found bacterial translocation to the kidney in the $\operatorname{E}$. coli $(n=3)$ and SF groups $(n=2)$, without statistical significance.

\section{Histology}

In the saline group, alveolar air expansion was found at a median of 2, i.e. between 26 and $50 \%$, ranging from 1-75\% (Fig. 3a). After SF \pm polymyxin treatment (Fig. 3c), this parameter increased to values above $25 \%$ with a median equivalent to $26-50 \%$ ( $p<0.05$ vs. saline). The $E$. coli group showed the most heterogenous alveolar air expansion giving a full range of semiquantitative assessment (median equivalent to 26-50\%; for example see Fig. 3b). Combined statistical analysis of both series showed that alveolar air expansion was significantly higher in animals treated with $\mathrm{SF} \pm$ polymyxin compared to saline controls or E. coli $(p<0.05)$. All 


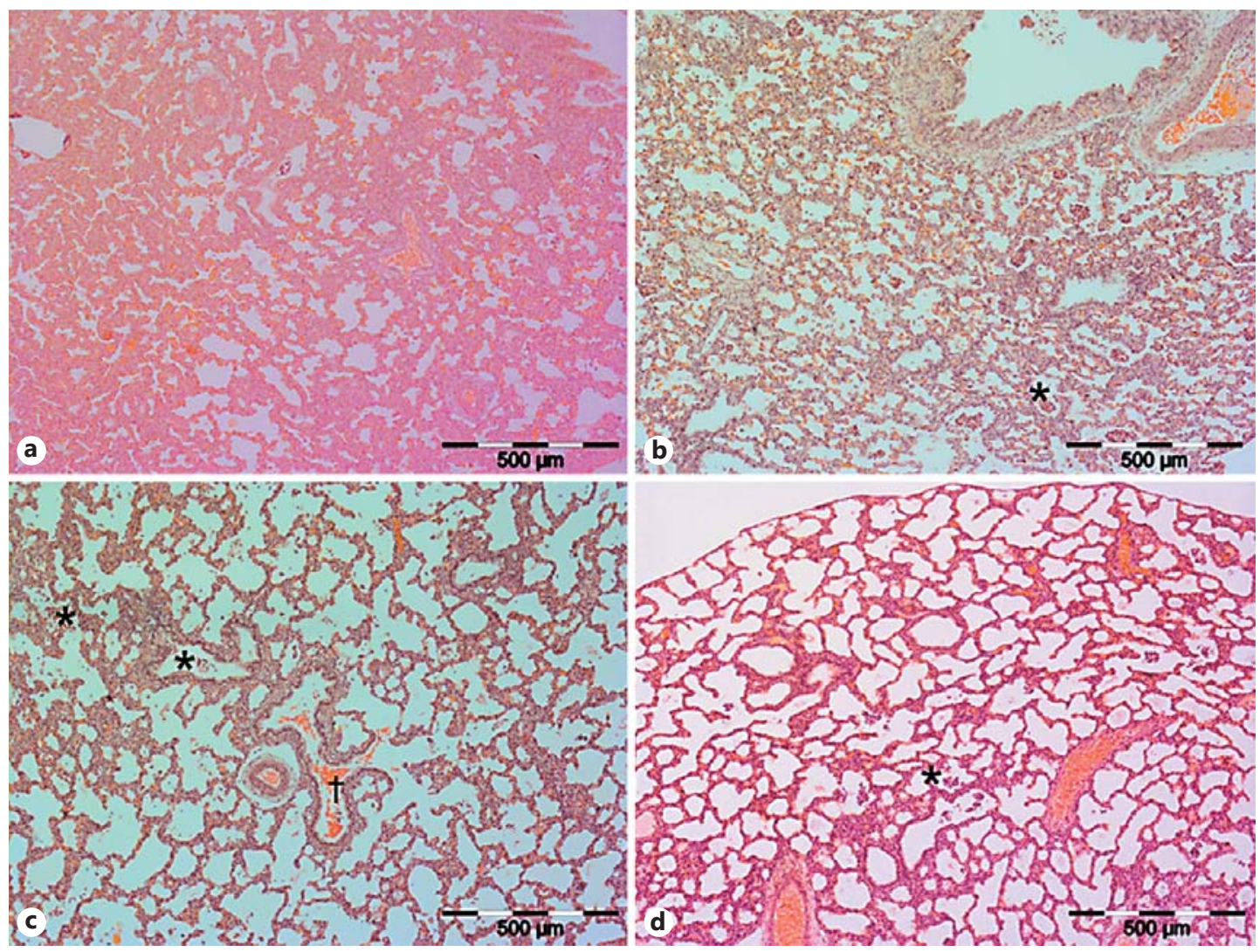

Fig. 3. Representative histologic lung sections. Representative images of transversal histologic lung sections stained with hematoxylin-eosin of neonatal rabbits treated with saline (a), Escherichia coli (b), surfactant and subsequently E. coli (d), or SF/polymyxin B 1\% followed by E. coli (c). Animals exposed to saline and only E. coli are nearly collapsed (a, b), whereas those treated with SF \pm polymyxin showed a higher degree of alveolar air expansion (c, d). Prominent histological inflammation including neutrophil influx and plugging of small airways $\left(^{*}\right.$ ) with conglomerates composed of neutrophils, bacteria, and erythrocytes was found in all treatment groups receiving bacteria (b-d). Additionally, prominent lung hemorrhage was found in SF/PxBtreated animals $\left({ }^{\dagger} \mathbf{c}\right)$.

animals inoculated with bacteria showed a high degree of pulmonary inflammation with no statistical differences between the groups (Fig. 3b-d). Inflammation was also found in the saline group, but at a significantly lower level compared to the E. coli group (Fig. 3a). Prominent pulmonary hemorrhage was found in the PxE versus PxB series at $30 \%$ in the E. coli group, $13 \%$ in the SF group, at $20 \%$ in the SF/PxE $1 \%$ group, and at $56 \%$ in the SF/PxB group $(p<0.05$ vs. SF/PxE 1\%). In the SF/PxE group of the PxE series, no severe pulmonary hemorrhage was found.

\section{Proinflammatory Markers}

A linear regression analysis of IL-8 levels versus survival time showed a significant deviation from a slope of zero for the saline group, but not for E.coli-treated animals. Thus, IL-8 increases significantly during ventilation in dead animals. Considering survivors, IL-8 was significantly increased in all animals treated with E. coli (median $2.2 \mathrm{ng} / \mathrm{g}$ lung; IQR: 1.6-4.0) versus the saline group (median $0.22 \mathrm{ng} / \mathrm{g}$ lung; IQR: $0.15-1.1 ; p<0.01$; Fig. 4). Values in groups treated with $E$. coli $\pm \mathrm{SF} \pm \mathrm{PxE}$ did not vary significantly from each other.

Kidney IL-8 levels of survivors showed no statistical differences. 
(C) 2017 The Author(s). Published by S. Karger AG, Basel www.karger.com/bmh

Stichtenoth et al.: Comparison of Polymyxin E and Polymyxin B as an Additive to Pulmonary Surfactant in Escherichia coli Pneumonia of Ventilated Neonatal Rabbits

Fig. 4. IL-8 levels in lung homogenates. IL-8 levels in homogenates of right lung of neonatal rabbits surviving 240 min of ventilation after initial treatment with surfactant (SF), SF plus different amounts of polymyxin $\mathrm{E}$ (SF/PxE 0.25\% and SF/PxE 1\%), or saline (saline or Echerichia coli group). With the exception of the saline group, all animals received Escherichia coli intratracheally at $15 \mathrm{~min}$ of ventilation. Scattergram of 5-7 repeated measurements. Bar $=$ median; ${ }^{* *} p<0.01$ vs. saline.

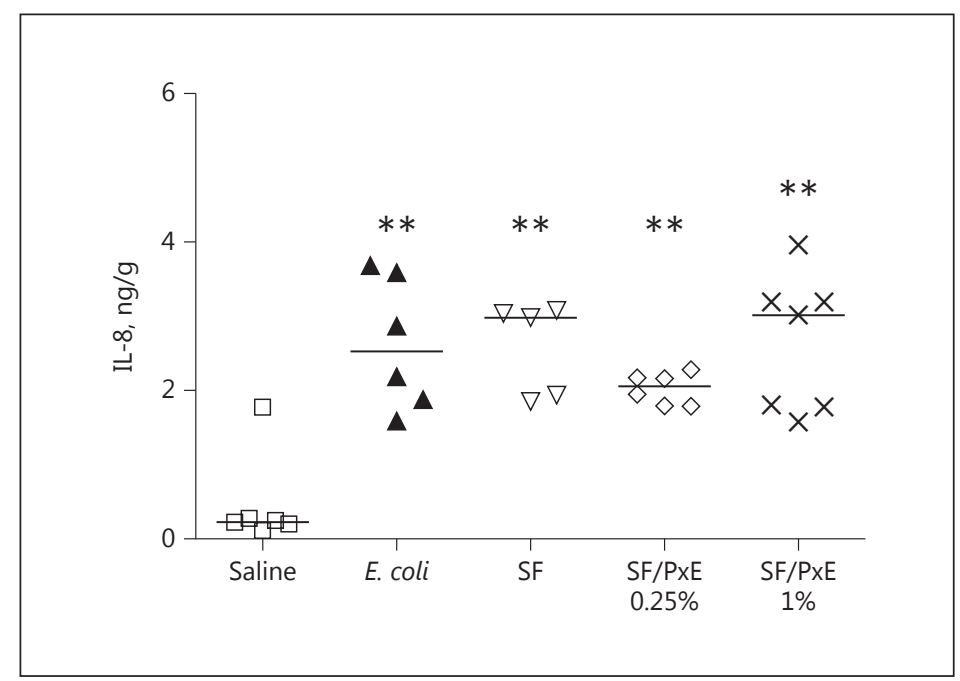

\section{Discussion}

This is the first study comparing intratracheal treatment of modified porcine SF + PxE versus $\mathrm{PxB}$ in an in vivo model of neonatal pneumonia.

We previously showed that addition of PxB to porcine SF in vitro resulted in maintained antimicrobial activity and improved surface activity [8]. In addition, PxB accelerates SF exocytosis in isolated cultured rat alveolar type II cells [9]. However, neither an improvement nor a deterioration of lung function could be found by comparing the compliance data of animals treated with only SF with those treated with SF plus admixed PxE or PxB.

For treatment of acute inflammatory lung injury, SF must have the greatest possible activity and resistance to inactivation [10]. Often, repeated high SF doses are needed to overcome inhibition as shown in premature neonates with culture-positive bronchoalveolar lavage [11]. The study underlines that high SF doses improve lung function in injury due to bacterial pneumonia: animals treated with SF or with SF + PxE each at $200 \mathrm{mg} / \mathrm{kg}$ had significantly increased compliance compared to the $E$. coli group. This is in line with the finding that treatment with $\mathrm{SF} \pm$ polymyxin resulted in significantly increased alveolar air expansion compared to saline- or E. coli-exposed animals.

Inoculation of bacteria resulted in severe inflammation of the lungs as confirmed by our histological findings and increased IL-8 levels. The binding property of administered polymyxin cannot reduce the lipopolysaccharide excess originating from Gram-negative bacteria. Thus, the detected IL-8 levels distinguish only between maximum and low inflammation. Inflammation may be attenuated and sepsis may be reduced in experimental pneumonia using different ventilation strategies, e.g. with high positive end-expiratory pressures [12, 13].

Compared to the E. coli and SF/PxB groups, a lower degree of pulmonary hemorrhage was found in animals treated with SF only or SF/PxE. This finding is surprising since we did not find any explanations for this in the literature.

In the present study, the addition of PxE or PxB to SF decreased the bacterial load of lungs, which agrees with previous studies on SF/PxB [14]. In a direct comparison, SF/PxE reduces the bacterial load more effectively than SF/PxB. In our study, we used polymyxin sulfate preparations. Sulfation and sulfomethylation may influence antibacterial activity [15] and membrane binding of PxE [16]. PxE sulfate appears to have a higher antibacterial activity compared to PxE (colistin-) methanesulfonate sodium, which presents a prodrug $[5,16]$. 
Thus, the PxE form used in the present study is more active than the form used clinically for inhalation, such as in cystic fibrosis. The high antimicrobiotic activity correlates to the applied polymyxin concentrations that, in lung homogenates, amount to $\sim 10-20 \mu \mathrm{g} / \mathrm{mL}$, which is 10 to 20 -fold of the minimal inhibitory concentrations of PxE $(=0.25-1 \mu \mathrm{g} / \mathrm{mL})$ or $\mathrm{PxB}(=0.25-2$ $\mu \mathrm{g} / \mathrm{mL}$ ) versus E. coli ATCC 25922 [17].

Recently, in vitro studies on bacterial growth found dose-dependent activity of mixtures composed of porcine SF/colistin-methanesulfonate sodium compared to only colistin-methanesulfonate sodium, which is found at low and disappears at higher concentrations of $\mathrm{PxE}$ in presence of $1 \mathrm{mg} / \mathrm{mL} \mathrm{SF}$ ( $\geq 64 \times$ minimal inhibitory concentration) [18]. Previous studies suggest a reduced PxE activity in the presence of tissue homogenates, lipid extracts, and phospholipids [19], a major constituent of pulmonary SF.

$\mathrm{SF}$ as a vehicle for drug delivery is an interesting concept when the pharmacological target is localized in the conducting airways. For antibiotic treatment, this could be during reduced innate or acquired immunity such as during premature birth, mechanical ventilation, chemotherapy, or chronic or acute pulmonary infections. Polymyxins exclusively target Gram-negative bacteria. Liposomal packing of polymyxins has been evaluated for inhalation and for systemic treatment, in order to reduce polymyxin toxicity [20]. Packed in SF, PxE presence in the conducting airways could be extended. At present, the pharmacokinetic parameters of SF/polymyxins such as distribution, half-life, and elimination remain unclear. Further, we do not know whether polymyxins spread along with the SF.

Differences between bacterial translocation to the kidney were found only in the "PxE series" but not in the "PxE versus PxB series," in which the kidney was washed additionally. Thus, bacterial load and differences regarding bacterial translocation might no longer be of significance.

The present study confirms that prophylactic intratracheal treatment with SF/PxE reduces pulmonary bacterial load in neonatal rabbit $E$. coli pneumonia. Compared to SF treatment alone, pulmonary compliance is maintained. The model used is suitable for rapid investigation of the early effects on inflammation, lung function, and bacterial load during short-time ventilation. Comparison between treatment with SF/PxB and SF/PxE favors the latter preparation. Further studies are needed to elucidate therapeutic treatment of SF/polymyxin mixtures and the significance of different PxE preparations.

\section{Disclosure Statement}

G.S. and T.C. received research grants from Chiesi. E.H. received speakers' honoraria, research grants, and worked as scientific advisor for Chiesi.

\section{Statement of Ethics}

Ethical permission was obtained from the local ethical board, Stockholms Norra djurförsöksetiska nämnd (ref. No. 316/12). 
2017 The Author(s). Published by S. Karger AG, Basel www.karger.com/bmh

Stichtenoth et al.: Comparison of Polymyxin E and Polymyxin B as an Additive to Pulmonary Surfactant in Escherichia coli Pneumonia of Ventilated Neonatal Rabbits

\section{References}

1 Simonsen KA, Anderson-Berry AL, Delair SF, Davies HD: Early-onset neonatal sepsis. Clin Microbiol Rev 2014; 27:21-47.

2 Benitz WE, Gould JB, Druzin ML: Risk factors for early-onset group B streptococcal sepsis: estimation of odds ratios by critical literature review. Pediatrics 1999;103:e77.

3 Gantert M, Been JV, Gavilanes AW, Garnier Y, Zimmermann LJ, Kramer BW: Chorioamnionitis: a multiorgan disease of the fetus? J Perinatol 2010;30:S21-S30.

-4 Starner TD, Agerberth B, Gudmundsson GH, McCray PB Jr: Expression and activity of beta-defensins and LL-37 in the developing human lung. J Immunol 2005;174:1608-1615.

-5 Velkov T, Roberts KD, Nation RL, Thompson PE, Li J: Pharmacology of polymyxins: new insights into an "old" class of antibiotics. Future Microbiol 2013;8:711-724.

-6 van't Veen A, Gommers D, Mouton JW, Kluytmans JA, Krijt EJ, Lachmann B: Exogenous pulmonary surfactant as a drug delivering agent: influence of antibiotics on surfactant activity. Br J Pharmacol 1996;118:593-598.

7 Haitsma JJ, Lachmann U, Lachmann B: Exogenous surfactant as a drug delivery agent. Adv Drug Deliv Rev 2001;47:197-207.

-8 Stichtenoth G, Jung P, Walter G, Johansson J, Robertson B, Curstedt T, Herting E: Polymyxin B/pulmonary surfactant mixtures have increased resistance to inactivation by meconium and reduce growth of Gramnegative bacteria in vitro. Pediatr Res 2006;59:407-411.

-9 Stichtenoth G, Herting E, Rüdiger M, Wemhöner A: Pulmonary surfactant preserves viability of alveolar type II cells exposed to polymyxin B in vitro. PLoS One 2013;8:e62105.

10 Willson DF, Notter RH: The future of exogenous surfactant therapy. Respir Care 2011;56:1369-1386.

11 Vento G, Tana M, Tirone C, Aurilia C, Lio A, Perelli S, Ricci C, Romagnoli C: Effectiveness of treatment with surfactant in premature infants with respiratory failure and pulmonary infection. Acta Biomed 2012;83(suppl 1):33-36.

-12 van Kaam AH, Lachmann RA, Herting E, De Jaegere A, van Iwaarden F, Noorduyn LA, Kok JH, Haitsma JJ, Lachmann B: Reducing atelectasis attenuates bacterial growth and translocation in experimental pneumonia. Am J Respir Crit Care Med 2004;169:1046-1053.

13 van Kaam AH, Lutter R, Lachmann RA, Haitsma JJ, Herting E, Snoek M, De Jaegere A, Kok JH, Lachmann B: Effect of ventilation strategy and surfactant on inflammation in experimental pneumonia. Eur Respir J 2005;26: 112-117.

14 Stichtenoth G, Linderholm B, Björkman MH, Walter G, Curstedt T, Herting E: Prophylactic intratracheal polymyxin B/surfactant prevents bacterial growth in neonatal Escherichia coli pneumonia of rabbits. Pediatr Res 2010;67:369-374

15 Li J, Turnidge J, Milne R, Nation RL, Coulthard K: In vitro pharmacodynamic properties of colistin and colistin methanesulfonate against Pseudomonas aeruginosa isolates from patients with cystic fibrosis. Antimicrob Agents Chemother 2001;45:781-785.

16 Craig WA, Kunin CM: Significance of serum protein and tissue binding of antimicrobial agents. Annu Rev Med 1976;27:287-300.

17 Jones RN, Anderegg TR, Swenson JM; Quality Control Working Group: Quality control guidelines for testing Gram-negative control strains with polymyxin B and colistin (polymyxin E) by standardized methods. J Clin Microbiol 2005;43:925-927.

18 Schwameis R, Erdogan-Yildirim Z, Manafi M, Zeitlinger MA, Strommer S, Sauermann R: Effect of pulmonary surfactant on antimicrobial activity in vitro. Antimicrob Agents Chemother 2013;57:5151-5154

19 Kunin CM: Binding of antibiotics to tissue homogenates. J Infect Dis 1970;121:55-64.

20 Wang D, Kong L, Wang J, He X, Li X, Xiao Y: Polymyxin E sulfate-loaded liposome for intravenous use: preparation, lyophilization, and toxicity assessment in vivo. PDA J Pharm Sci Technol 2009;63:159-167. 